\section{Case Reports in Ophthalmology}

\title{
Airbag-Associated Severe Blunt Eye Injury Causes Choroidal Rupture and Retinal Hemorrhage: A Case Report
}

\author{
Shih Hao Wang ${ }^{a}$ Chen Chee Lim ${ }^{b}$ Yu Ti Teng ${ }^{a}$ \\ aDepartment of Ophthalmology, National Cheng Kung University Hospital, College of \\ Medicine, National Cheng Kung University, Tainan, Taiwan; ${ }^{b}$ Department of Medicine, \\ National Cheng Kung University Hospital, Tainan, Taiwan
}

\section{Keywords}

Airbags $\cdot$ Choroidal rupture $\cdot$ Intravitreal sulfur hexafluoride $\cdot$ Intravitreal bevacizumab

\begin{abstract}
A case of choroidal rupture caused by airbag-associated blunt eye trauma and complicated with massive subretinal hemorrhage and vitreous hemorrhage that was successfully treated with intravitreal injection of expansile gas and bevacizumab is presented. A 53 -year-old man suffered from loss of vision in his right eye due to blunt eye trauma by a safety airbag after a traffic accident. On initial examination, the patient had no light perception in his right eye. Dilated ophthalmoscopy revealed massive subretinal hemorrhage with macular invasion and faint vitreous hemorrhage. We performed intravitreal injection of pure sulfur hexafluoride twice for displacement, after which visual acuity improved to 0.03 . For persistent subretinal hemorrhage and suspicion of choroidal neovascularization (CNV), intravitreal bevacizumab $(1.25 \mathrm{mg} / 0.05 \mathrm{~mL})$ injection was administered. After 3 weeks, the visual acuity of his right eye recovered to 0.4 . For early-stage choroidal rupture-induced subretinal hemorrhage and complications of suspected CNV, intravitreal injection of expandable gas and intraocular injection of antiangiogenesis drugs seem to be an effective treatment.
\end{abstract}

(C) 2017 The Author(s)

Published by S. Karger AG, Basel 


\section{Case Reports in Ophthalmology}

\section{Introduction}

Choroidal rupture, first defined by von Graefe in 1854, is a type of closed-globe injury caused by blunt eye trauma that results in a rupture of the Bruch membrane and the retinal pigment epithelium layer due to anterior-posterior compression and horizontal expansion of the eye. During the trauma caused by the injury, the relatively strong collagen-fortified sclera and the relatively flexible retina are not easily ruptured, but the relatively inflexible retinal pigment epithelium layer, Bruch's membrane, and the neighboring choriocapillaris can be ruptured more easily. Choroidal ruptures can be divided into direct and indirect types. The indirect type is more common; the rupture is often located at the side opposite the impact. Approximately $5-10 \%$ of all blunt eye injuries are accompanied by indirect choroidal rupture [1]. However, indirect choroidal rupture generally occurs at the posterior pole, forming a shape that is crescentic and concentric to the optic nerve. If the location of the choroidal rupture affects the macular area or is below the fovea, it will lead to relatively poor visual outcome [2].

Airbags are inflatable devices that are designed to reduce car accident-related mortality and morbidity. Nevertheless, due to the widespread use of airbags, incidents of eye trauma have been reported in recent years, although largely in the form of case reports. Trauma intensity varies from mild to severe, ranging from injuries of the anterior segment to the posterior segment, including corneal abrasion, hyphema, eyelid laceration, traumatic iritis, iris tear, cataract, angle recession, corneal or scleral laceration, chemical keratitis, dislocated lens, orbital fracture, facial nerve palsy, vitreous/retinal hemorrhage, retinal tear or detachment, commotio retinae, macular hole, choroidal rupture, traumatic maculopathy, traumatic optic neuropathy, retinitis sclopetaria, and LASIK flap folds [3].

This case study reports on a patient who suffered from choroidal rupture with severe subretinal hemorrhage that was caused by rapidly inflating airbags during a car accident.

\section{Case Report}

The work described has been carried out in accordance with The Code of Ethics of the World Medical Association (Declaration of Helsinki). Informed consent was obtained for experimentation.

The patient was a 53-year-old man in good health without any major illnesses. He claimed that in a recent car accident, which had occurred 3 days before he reported to the clinic, his right eye was hit by a suddenly inflating airbag. In addition to severe pain, he suffered from a sudden-onset loss of vision. Based on the patient's description, his car was involved in a full frontal collision with a roadside sign as he was making a left turn. He was not wearing his seatbelt and was driving at a speed of $50 \mathrm{~km} / \mathrm{h}$. The first clinical examination revealed that his right eye was not perceptive to light. The visual acuity of his left eye was 0.8. The intraocular pressure (IOP) of his right and left eye was 12 and $15 \mathrm{~mm} \mathrm{Hg}$, respectively. Slit lamp examination revealed that the anterior segment was largely normal and did not exhibit corneal or scleral laceration. Dilated fundus examination revealed that his right eye had massive subretinal hemorrhage around the optic disc with macular involvement in addition to vitreous hemorrhage, while his left eye was normal. Although the resolution was poor due to vitreous hemorrhage, optical coherence tomography (OCT) revealed a hint of macular edema with subretinal fluid with a central retinal thickness of $436 \mu \mathrm{m}$ (Fig. 1). After a week of observation, the right eye still had no perception to light. Therefore, the patient 


\section{Case Reports in Ophthalmology}

Wang et al.: Airbag-Associated Severe Blunt Eye Injury Causes Choroidal Rupture and Retinal Hemorrhage: A Case Report

was advised to have an intravitreal injection of $0.3 \mathrm{~mL}$ sulfur hexafluoride (SF6) in the right eye. The patient agreed, and the injection was administered with him in the prone position. Four days after the injection, vision in the right eye had improved. The patient could recognize hand motion within a distance of $60 \mathrm{~cm}$. The IOP was $12 \mathrm{~mm} \mathrm{Hg}$. Dilated fundus examination revealed significantly reduced subretinal macular hemorrhage, which was pushed to the periphery of the retina. It was also observed that the location of the choroidal rupture was on the superior and the temporal side of the macula. OCT results suggested that the macular edema had significantly reduced, and the central retinal thickness had decreased to $278 \mu \mathrm{m}$, but it still contained some subretinal fluid. Since the intraocular gas had nearly disappeared, it was subsequently recommended that the patient receive a second intravitreal injection of $0.3 \mathrm{~mL} \mathrm{SF} 6$ in the right eye. One week after the injection, visual acuity of the right eye had improved to 0.03 , but the subretinal hemorrhage and the vitreous hemorrhage conditions had not improved at all (Fig. 2), while the central retinal thickness had slightly increased to $319 \mu \mathrm{m}$.

Since it was possible that a secondary choroidal neovascularization (CNV) had occurred, after fully informing the patient of the potential benefits and risks, we scheduled the patient for immediate intravitreal injection of $1.25 \mathrm{mg} / 0.05 \mathrm{~mL}$ bevacizumab in the right eye. Three weeks after the injection, the corrected visual acuity of the right eye had improved to 0.4, with only small amounts of residual subretinal hemorrhage at the superior, inferior, and nasal side of the eye. Vitreous hemorrhage had almost entirely disappeared as well. Fundus examination revealed a visible scar caused by the choroidal rupture. OCT results showed a complete absence of subretinal fluid, and the central retinal thickness had decreased to 210 $\mu \mathrm{m}$. The location of the choroidal rupture was observed on fluorescein angiography, and dye leakage was not observed during the early or late phase (Fig. 3).

\section{Discussion}

Airbags are soft inflatable pouches that are installed in automobiles. In the event of a collision or upon detecting a sudden deceleration of the vehicle, in order to prevent the head, face, or body of the passengers from making direct contact with the steering wheel, dashboard, or windshield, airbags will rapidly inflate within one-tenth of a second to reduce the severity of the bodily harm and to prevent passengers from being ejected from the seats, thus also preventing secondary injuries. Airbags are therefore designed to prevent accidentrelated casualties.

Pearlman et al. [3] stated that, between 1991 and 2000, there were a total of 263 incidents of airbag-related eye injuries in 101 patients. From an anatomical perspective, the type of eye injury with the highest incidence was corneal injuries, which accounted for $21.6 \%$ of all eye injuries and included corneal epithelial defects and chemical keratitis. Hyphema had the second highest incidence, accounting for $17.1 \%$ of all injuries, followed by vitreous and retinal hemorrhage at $9.9 \%$, retinal tears and retinal detachment at $5.7 \%$, commotio retinae at $5.3 \%$, and angle recession at $4.2 \%$. There were only 3 incidents of airbag-related choroidal ruptures, accounting for $1.1 \%$, which is generally considered to be a rare form of complication. There were 11 cases of open globe injuries, such as scleral and/or corneal laceration, accounting for $4.2 \%$ of the total. Rupture of the eyeball is considered a severe complication; therefore, patients who suffer rupture often have a poor visual prognosis. Despite the fact that multiple possible airbag-related eye complications do occur, $45 \%$ of major accidents resulted in eyeball rupture even before airbags were widely used. It is therefore accurate to 


\section{Case Reports in Ophthalmology}

Wang et al.: Airbag-Associated Severe Blunt Eye Injury Causes Choroidal Rupture and Retinal Hemorrhage: A Case Report

suggest that airbags can significantly reduce the severity of accident-related ocular trauma [4].

The main mechanism of airbag-related eye injuries includes blunt ocular trauma from the impact of the inflating airbag and chemical keratitis after exposure to the alkaline sodium azide gas that is released during the deflation of the airbag [5]. Corneal epithelial defect or hyphema of the anterior segment of the eye are usually self-limiting. However, if the posterior segment is affected, such as in retinal detachment, choroidal rupture, macular hole, and traumatic optic neuropathy, it may result in a permanent loss of vision. In our case, the patient was found to suffer from choroidal rupture with massive subretinal and vitreous hemorrhage after a blunt injury to the right eye. He was not found to have hyphema, lens shift, or corneal damage. In addition, we also observed that the IOP of the patient was normal, and there was neither ocular hypotony nor hypotony maculopathy. In the subsequent follow-up examinations, complications such as retinal tear, retinal detachment, or macular hole were also not observed.

Yang et al. [6] have suggested that Asians tend to have a higher chance of incurring airbag-related eye injuries. This may be related to the orbital anatomical structure of Asians, as a shallow orbital socket and the less obvious orbital rim may allow airbags to make direct contact with the eyeball. In addition, compared to Caucasians, Asians tend to have a smaller stature. Therefore, they are more likely to be closer to the steering wheel and the airbag, and are thus more likely to be hit by a suddenly inflating airbag. Furthermore, if the seatbelt is worn while driving, it may reduce the force with which the eyeball is hit and, therefore, prevent subsequent complications. It would be interesting to evaluate whether there is a difference in the severity of airbag-related eye injuries for Asians driving European-, Japanese-, Korean-, and Taiwanese-made vehicles. Is there a need to develop airbags tailored for Asians? Additional research is needed on this subject.

For the treatment of choroidal rupture, there are currently no medications or surgical procedures available that are particularly effective. However, a few case reports have suggested that the intravitreal injection of expandable gases, for example, SF6 or C3F8 (perfluoropropane), along with tissue plasminogen activator can have a positive therapeutic effect on treating choroidal ruptures with newly incurred subretinal hemorrhage [7]. The therapy has also been shown to be effective in treating subretinal hemorrhage that is complicated by wet age-related macular degeneration [8]. In addition, expandable gas can also be injected intravitreally after performing macular hole and retinal detachment surgery to increase the success rate of the procedure [9]. Currently, although there are no large-scale studies on the treatment of choroidal rupture-associated subretinal hemorrhage, we employed the therapy that was documented for the treatment of wet age-related macular degeneration-associated retinal hemorrhage to treat our patient, in the hope that the expandable gas in combination with the prone position would push the hemorrhage beneath the macula to the periphery of the eye in order to restore the patient's central vision. The result of the procedure was positive.

In addition to causing complicated hemorrhage, choroidal rupture can result in CNV in about $20 \%$ of the cases, usually next to the scar that has resulted from the previous choroidal rupture. CNV, if left untreated, may lead to additional retinal hemorrhage or cause fibrosis and result in loss of vision [10].

Currently, many studies have stated that injection of an antivascular endothelial growth factor agent is significantly effective in treating CNV caused by wet age-related macular degeneration, diabetic retinal edema, retinopathy of prematurity, and intraocular inflammation or infection. In addition, the procedure of intravitreal injection is very safe, time-saving, and 


\section{Case Reports in Ophthalmology}

\section{\begin{tabular}{l|l}
\hline DOI: $10.1159 / 000452652$ & (C) 2017 The Author(s). Published by S. Karger AG, Basel
\end{tabular}} www.karger.com/cop

Wang et al.: Airbag-Associated Severe Blunt Eye Injury Causes Choroidal Rupture and Retinal Hemorrhage: A Case Report

inexpensive, and most patients can be treated on an outpatient basis. In 2004, bevacizumab (Avastin) was approved by the Food and Drug Administration (FDA) for the treatment of metastatic colorectal cancer, but intravitreal injection in ophthalmology is still considered an off-label use of the medication. There are only a handful of studies on choroidal ruptureinduced CNV [11], and currently there is a lack of large-scale studies to confirm the efficacy of antiangiogenesis drugs. In our case, we employed the therapeutic dose used to treat wet age-related macular degeneration for our patient, who was given an intravitreal injection of $1.25 \mathrm{mg} / 0.05 \mathrm{~mL}$ bevacizumab once. Although at the beginning, fluorescent angiography did not provide any evidence, fortunately, the visual prognosis of the patient was satisfactory. However, CNV may still occur several years after the injury [10], and, therefore, regular follow-ups over a long period of time are necessary to observe and track the progress of the disease. In addition, there have been several documentations of complications arising from intravitreal injections, such as retinal tear and detachment, exogenous endophthalmitis, ocular hypertension, and vitreous hemorrhage [12]. Literature has indicated that intravitreal injection of bevacizumab may also increase the risk of cardiovascular and cerebrovascular complications, for example, acute blood pressure elevation or myocardial infarction [13].

According to the statistics published by Taiwan's National Police Agency [14], in 2014, 307,482 incidences of traffic accidents were documented, which resulted in approximately 2,612 cases of fatalities and 412,436 cases of injuries. These accidents caused severe injuries and were a substantial burden to society in terms of cost. In order to reduce the rate of accidents, important issues such as drafting comprehensive transport policies and regulations and educating the public on the importance of obeying traffic rules also need to be addressed.

The widespread use of airbags can indeed reduce the occurrence of accident-related casualties, but it can also result in relatively more cases of airbag-related eye trauma that can cause complications and ruptures of the posterior segment of the eye, leading to severe cases of loss of vision. Therefore, a timely diagnosis, interventional therapy, and follow-up tracking are very important and necessary. Large-scale studies would need to be conducted on the treatment of choroidal rupture in order to determine the best therapeutic approach. However, for early-stage choroidal rupture-induced subretinal hemorrhage and the complications of suspected CNV, intravitreal injection of expandable gas with the patient in the prone position, in addition to intraocular injection of antiangiogenesis drugs, seems to be an effective treatment.

\section{Statement of Ethics}

Written informed consent was obtained from the patient for the publication of this case report and any accompanying images.

\section{Disclosure Statement}

The authors declare that there is no conflict of interest regarding the publication of this paper. No funding was received for this work. 
Wang et al.: Airbag-Associated Severe Blunt Eye Injury Causes Choroidal Rupture and Retinal Hemorrhage: A Case Report

\section{References}

-1 Eagling EM: Ocular damage after blunt trauma to the eye. Its relationship to the nature of the injury. Br J Ophthalmol 1974;58:126-140.

-2 Wood CM, Richardson J: Indirect choroidal ruptures: aetiological factors, patterns of ocular damage, and final visual outcome. Br J Ophthalmol 1990;74:208-211.

-3 Pearlman JA, Au Eong KG, Kuhn F, Pieramici DJ: Airbags and eye injuries: epidemiology, spectrum of injury, and analysis of risk factors. Surv Ophthalmol 2001;46:234-242.

4 Kuhn F, Morris R, Witherspoon CD: Eye injury and the air bag. Curr Opin Ophthalmol 1995;6:38-44.

-5 Smally AJ, Binzer A, Dolin S, Viano D: Alkaline chemical keratitis: eye injury from airbags. Ann Emerg Med 1992;21:1400-1402.

-6 Yang CS, Chou TF, Liu JH, Hsu WM: Air bag associated posterior segment ocular trauma. J Chin Med Assoc 2004;67:425-431.

7 Holland D, Wiechens B: Intravitreal r-TPA and gas injection in traumatic submacular hemorrhage. Ophthalmologica 2004;218:64-69.

8 Meyer CH, Scholl HP, Eter N, Helb H-M, Holz FG: Combined treatment of acute subretinal haemorrhages with intravitreal recombined tissue plasminogen activator, expansile gas and bevacizumab: a retrospective pilot study. Acta Ophthalmol 2008;86:490-494.

-9 Wendel RT, Patel AC, Kelly NE, Salzano TC, Wells JW, Novack GD: Vitreous surgery for macular holes. Ophthalmology 1993;100:1671-1676.

10 Secrétan M, Sickenberg M, Zografos L, Piguet B: Morphometric characteristics of traumatic choroidal ruptures associated with neovascularization. Retina 1998;18:62-66.

-11 Yadav NK, Bharghav M, Vasudha K, Shetty KB: Choroidal neovascular membrane complicating traumatic choroidal rupture managed by intravitreal bevacizumab. Eye (Lond) 2009;23:1872-1873.

-12 Jager RD, Aiello LP, Patel SC, Cunningham ET Jr: Risks of intravitreous injection: a comprehensive review. Retina 2004;24:676-698.

13 Wu L, Martínez-Castellanos MA, Quiroz-Mercado H, Arevalo JF, Berrocal MH, Farah ME, Maia M, Roca JA, Rodriguez FJ; Pan American Collaborative Retina Group (PACORES): Twelve-month safety of intravitreal injections of bevacizumab (Avastin): results of the Pan-American Collaborative Retina Study Group (PACORES). Graefes Arch Clin Exp Ophthalmol 2008;246:81-87.

14 Taiwan National Police Agency, Ministry of the Interior. http://www.npa.gov.tw/ (accessed April 16, 2015).
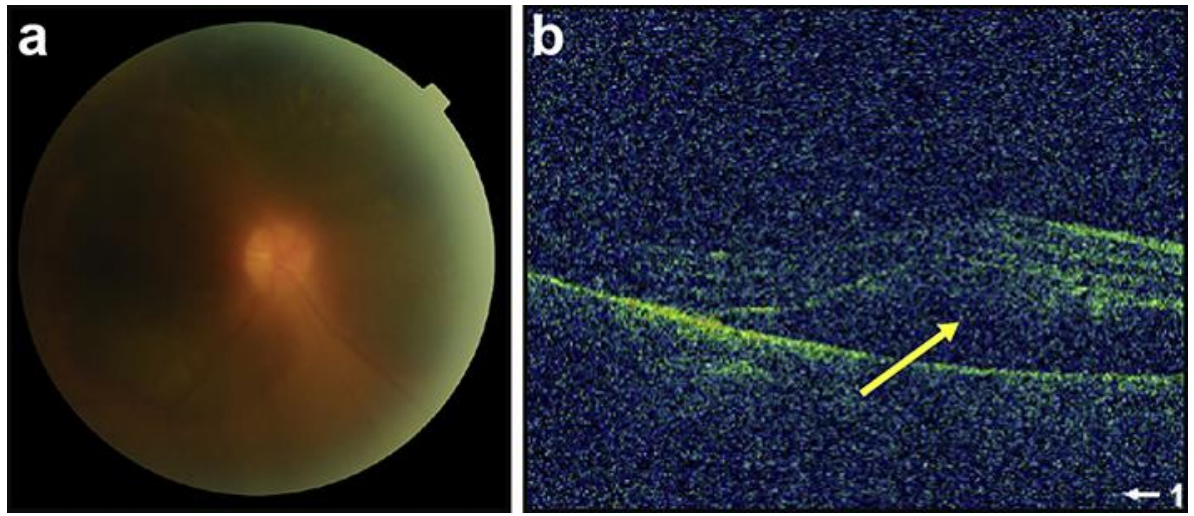

Fig. 1. The fundus and the OCT during the initial patient diagnosis. a The patient's right eye: massive subretinal hemorrhage can be seen around the optic disc with macular involvement accompanied by vitreous hemorrhage. b Subretinal fluid (arrow) and macular edema are visible; the central retinal thickness increased to $436 \mu \mathrm{m}$. 


\section{Case Reports in Ophthalmology} www.karger.com/cop

Wang et al.: Airbag-Associated Severe Blunt Eye Injury Causes Choroidal Rupture and Retinal Hemorrhage: A Case Report
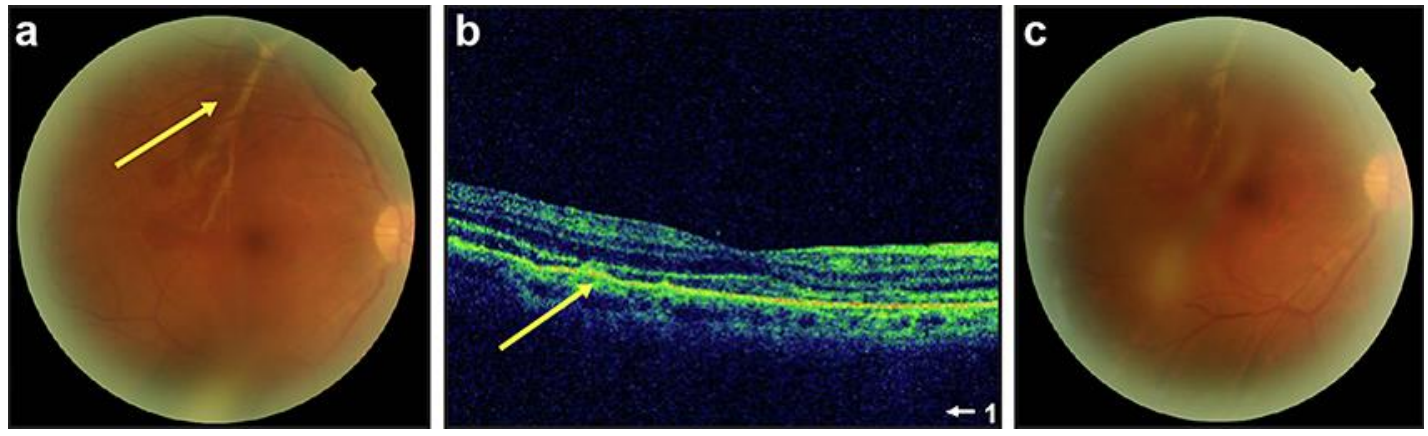

Fig. 2. a After 2 times intravitreal injection (IVI) of SF6, subretinal hemorrhage in the fundus has diminished significantly. The scar resulting from the choroidal rupture can be seen (arrow); a large amount of hemorrhage is still visible surrounding the scar, and vitreous hemorrhages decreased slightly compared to before. b OCT after the first IVI of SF6: macular edema has improved significantly, only some subretinal fluid is visible on the temporal side of the fovea. The scar from the choroidal rupture (arrow) can be seen; central retinal thickness has reduced to $278 \mu \mathrm{m}$. c After the second IVI of SF6, no significant changes can be observed in the fundus; subretinal and vitreous hemorrhage is still visible around the scar. Central retinal thickness in OCT has slightly increased to $319 \mu \mathrm{m}$. 


\section{Case Reports in Ophthalmology}
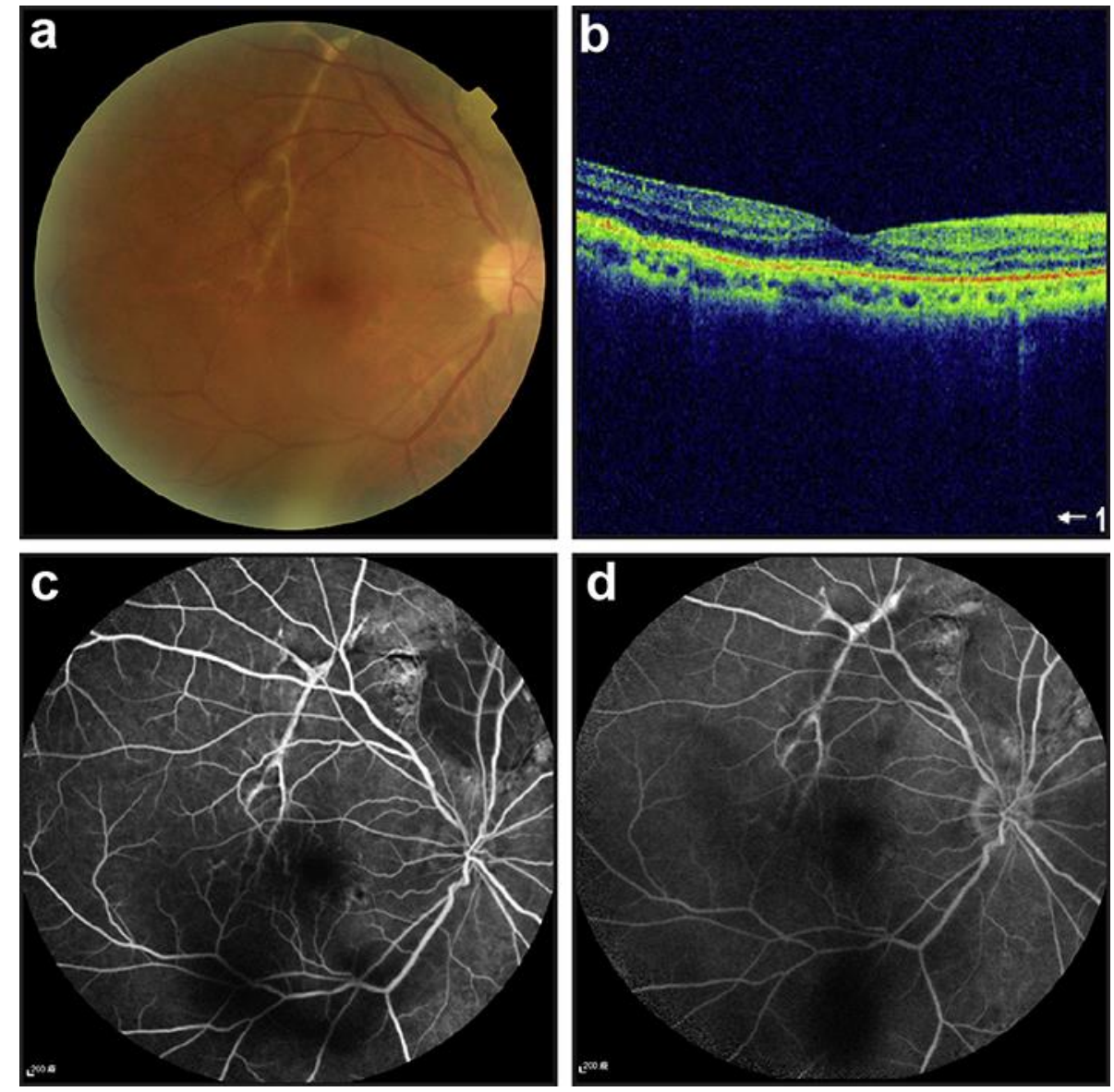

Fig. 3. a After the intravitreal injection (IVI) of Avastin, the scar from the choroidal rupture can be observed at the temporal side of the macular area; subretinal and vitreous hemorrhage has nearly disappeared. $\mathbf{b}$ After the IVI of Avastin, the subretinal fluid disappeared entirely; CRT is $210 \mu \mathrm{m}$, and macular edema is absent. c Early phase of fluorescein angiography after the IVI of Avastin. $\mathbf{d}$ Late phase of fluorescein angiography after the IVI of Avastin; obvious dye leakage cannot be observed. 